\title{
USE OF SMALL INTESTINE SUBMUCOSA AS URETERAL ALLOGRAFT IN PIGS
}

\author{
FERNANDO H. GRECA, LUCIA NORONHA, MARCELO BENDHACK, ANDRÉ FERES, \\ ANDRÉA SOCCOL, JOÃO R. DUDA \\ Department of Experimental Surgery, Pontifical Catholic University of Parana, Curitiba, Parana, Brazil
}

\begin{abstract}
Purpose: The aim of the present study was to evaluate the biocompatibility of small intestine submucosa (SIS) in the reconstruction of the ureter in swine.

Materials and Methods: An experimental study was performed in 10 half-breed pigs weighing between 20 and $30 \mathrm{~K}$, in which a previously prepared segment of SIS measuring approximately 2.0 $\mathrm{cm}$ was implanted in the upper third part of the right ureter.

Results: Of the 10 operated animals, one died 14 days after the surgery due to a dehiscence on the suture line of the implanted graft. The remaining 9 animals were submitted to ultrasound examination of the urinary tract and were sacrificed on the 40th postoperative day. The macroscopic evaluation showed no calculus, incrustation, fistula, abscesses or adhesions in the ureters with the graft. Microscopic evaluation with hematoxylin-eosin and Sirius red showed in the experimental area (graft) the presence of urothelium in $100 \%$ of the cases, collagen in $100 \%$ of the cases, and smooth muscle layer in $87.5 \%$ of the animals. In the area adjacent to the graft (proximal and distal), we observed $92.86 \%$ of urothelium, $42.86 \%$ of collagen and $71.43 \%$ of smooth muscle. In the contralateral ureter, it was found $100 \%$ of urothelium and smooth muscle and just $11.11 \%$ of collagen. The microscopic analysis of the kidneys whose ureters received the graft of SIS evidenced congestion in $55.55 \%$, pelvic edema in $66.66 \%$ and interstitial nephritis in $77.78 \%$. Hydronephrosis was present in $33.33 \%$ and chronic pyelonephritis in $44 \%$. Only 1 animal presented total absence of glomerulus in the renal parenchyma.

Conclusion: The SIS graft behaved as a biological tissue support, allowing the regeneration of the urothelium and smooth muscle grow, despite of chronic inflammatory process.
\end{abstract}

Key words: small intestine; submucosa; ureter; graft; swine

Int Braz J Urol. 2004; 30: 327-35

\section{INTRODUCTION}

Ureteral reconstruction represents a great challenge to the urologist, especially if a long segment of ureter is involved. Per decades, surgeons look for alternative ways to repair and to reconstruct the ureter, using different materials and techniques (1-3).

The current options for treatment are ureteroureterostomy, ureteroneocistostomy with the psoas hitch, transureterostomy, Boari flap and renal mobilization. Therefore, when a long ureteral segment is lost, other options are used such as permanent percutaneous nephrostomy, auto transplantation or simple nephrectomy $(2,3)$.

Another procedure that is being widely used at present in ureteral surgeries is the endoscopic treatment. However, this technique is not widely accepted and it is currently limited to patients with moderate strictures $(4,5)$.

Among the structures used for ureteral reconstruction are the appendix, veins (as the umbilical vein), arteries, Fallopian tube, skin grafts, 
stomach and peritoneum, even though with limited success. Alloplastic materials, such as cobaltchromium, polyvinyl chloride, polyethylene, polytetrafluoethylene, silver, silicon rubber, polyester, collagen and polyglactin have been investigated and the results were disappointing (rejection, stone formation, migration, urinary leak, anastomotic stricture and hydronephrosis with renal loss) $(1,2,6-8)$.

Results from numerous pre-clinical studies have demonstrated that SIS is capable to inducing host tissue proliferation and replacement when implanted in various sites. In the urinary tract, the SIS has been shown as a promising graft (9-19). It induce host tissue proliferation and replacement. Studies on the composition of SIS revealed water content of approximately $90 \%$. The protein content consists primarily of collagens, type I, III and V. The threedimensional matrix provides a suitable structural environment for the resident cells of a tissue. The glycoproteins, proteoglycans and glycosaminoglycans help the cell to attach and settle within the matrix. This property contributes to the re-population of the matrix and cell migration, proliferation and differentiation (20). Another component of the SIS biomaterial, critical to its mechanism of action is the growth factor content (21). They stimulate cell division, migration and differentiation. Among the growth factor found in the SIS membrane are fibroblasts growth factor-2, transforming growth factor $\beta$ and vascular endothelial growth factor $(22,23)$. Several studies have demonstrated resistance to bacterial infection and lack of adverse immunological reaction, probably due to the acellular condition and significant collagen composition, which is highly preserved across the species. The immunological response is restricted to the Th2 pathway, which may allow acceptance and remodeling of graft tissue (12).

The aim of the present study is to evaluate the SIS biocompatibility when used as a graft for ureter repair.

\section{MATERIALS AND METHODS}

An experimental study was accomplished in 10 half-breed pigs weighing between 20 and $30 \mathrm{Kg}$.
The protocol was approved by Institutional Animal Care and Use Committee of our Institution.

The small intestine was harvested from pigs sacrificed at a slaughterhouse and the SIS was prepared in the laboratory of Experimental Surgery of the Pontifical Catholic University of Parana. The tunica mucosa was mechanically removed from the inner surface and the serosa and tunica muscularis were mechanically removed from the outer surface. This procedure produces a thin translucent graft composed mainly of the submucosa layer of the intestinal wall. The membrane obtained was immediately placed in a solution containing neomycin $10 \%$ and normal saline solution, and stored at $5^{\circ} \mathrm{C}$ for 12 hours. A small segment of the membrane was then wrapped up in a catheter in order to produce a fine straw of the submucosa.

Under general anesthesia, a midline abdominal incision was made in the pig. The retroperitoneum was accessed. A segment of $2 \mathrm{~cm}$ length of the upper right ureter, involving two-thirds of its diameter was removed, parallel to the ureteral axis. A 4F pediatric nasogastric tube was inserted into the ureter through the created gap. A submucosa straw wrapped up the catheter and was sutured at the cut edges of the ureter with an uninterrupted 5-0 catgut suture (Figure-1).

No procedures were performed in the left ureter, which was used as a control. On the 30th

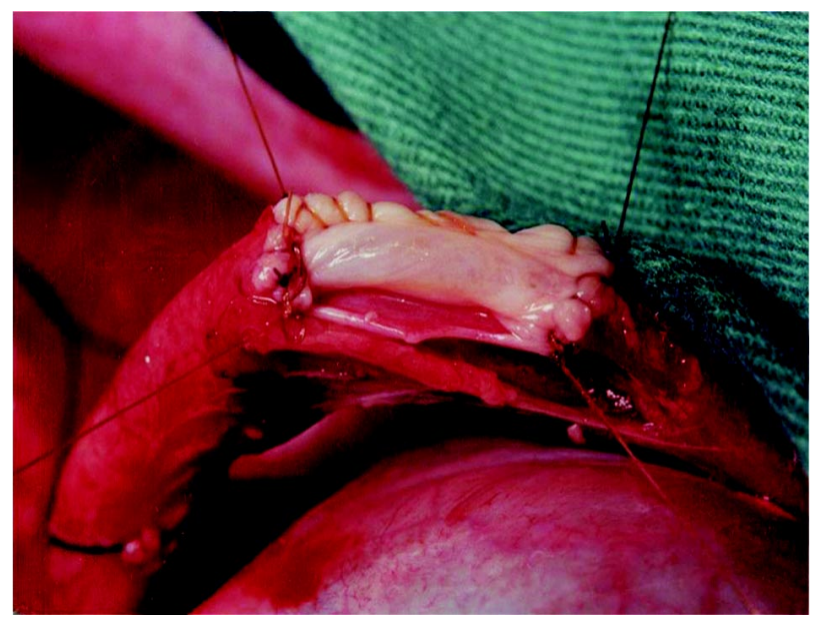

Figure 1 - Small intestine submucosa graft sutured in the cut edges of the right ureter. 
postoperative day, all animals were submitted to an ultrasound examination of the urinary tract and on the 40th postoperative day the pigs were sacrificed for removal of both kidneys and both ureters. The presence of adhesions, fistulas and abscesses were analyzed. The diameter of the upper third of the left ureter was measured as well as the diameter of the right ureter, in the site where the submucosa graft was placed and $1 \mathrm{~cm}$ proximal and $1 \mathrm{~cm}$ distal to it.

The site of the graft was named experimental area. The 2 areas adjacent to the graft were named referential areas. The correspondent areas of the left ureter were named control areas.

Microscopic evaluations - A sample of the experimental areas, the referential areas and the control areas were fixed in a 5\% formalin solution. The paraffin embedded tissue blocks were sectioned and stained with hematoxylin-eosin to evaluate the inflammatory reaction, the urothelium, collagen and muscle ingrowth. It was possible to quantify the inflammatory reaction into acute or chronic through a score table (Table-1).

The variables congestion, edema and neutrophils characterize an acute inflammatory process. The presence of granulation tissue, fibrosis and mononuclear cells characterize a chronic inflammatory phase. Thus, the results between -9 and -1 were considered as acute inflammatory phase, between -1 and +1 as absence of inflammatory process, and between +1 and +9 , chronic inflammatory phase.

The Sirius red staining was used to evaluate the collagen and muscle densitometry through Image Pro Plus Software. Under optical Zeiss microscope, with a magnification of 100X, 6 areas of the specimen were selected. The images were captured by a digital video camera and processed by a video-board in a personal computer. As the collagen and muscle can be stained in different colors, it was possible to quantify the amount of each component.

Both kidneys were also evaluated microscopically. The specimens were also stained with hematoxylin-eosin to assess the congestion, pelvic edema, interstitial nephritis, hydronephrosis, chronic pyelonephritis and glomerular absence.

Statistical analysis - The data were presented as mean \pm standard deviation, median or frequencies, when appropriate. Comparisons of smooth muscle and collagen percentage between groups were performed using the t-test for paired observations. Friedman test and Wilcoxon test were used to determine significant differences among groups for the inflammatory reaction. Comparisons between groups for nominal variables were made with binomial test. The tests were 2 -sided and p-values less than 0.05 were considered significant.

\section{RESULTS}

Of the 10 animals submitted to SIS graft implantation on the ureter, 1 died 14 days after surgery due to a dehiscence of the anastomosis. The ultrasound evaluation of the urinary tract, performed on the 30th post-operative day, showed a dilatation of the right renal pelvis in 2 pigs $(22.22 \%)$ in relation to the left renal pelvis. It was not possible to accomplish a reliable ultrasound evaluation of both ureters.

Table 1 - Variables used to obtain the final score.

\begin{tabular}{llcccc}
\hline Inflammatory process & Variables & Absence & Discreet & Moderate & Accentuated \\
\hline Acute - & Neutrophils & 0 & -1 & -2 & -3 \\
& Edema & 0 & -1 & -2 & -3 \\
& Congestion & 0 & -1 & -2 & -3 \\
Chronic + & & & & 3 \\
& Monocytes & 0 & 1 & 2 & 3 \\
& Granulation tissue & 0 & 1 & 2 & 3 \\
\hline
\end{tabular}


The macroscopic evaluation of the ureters showed no fistula, incrustation or abscess. The measurements of diameter showed partial stenosis in one animal and severe stenosis in another one. Table2 shows the mean diameter of the right ureter in the site of the graft and $1 \mathrm{~cm}$ above and bellow this area, as well as the measure of the correspondent area of the left ureter.

All measured diameters of the right proximal ureter were significantly greater than the diameter of the correspondent area of the left ureter. No statistically significant difference was found in the diameter of the right proximal ureter when compared to the graft site.

The histological analysis of the specimens stained with hematoxylin-eosin showed in the experimental area (area where the graft was placed) the presence of urothelium in $100 \%$ of the cases, collagen in $100 \%$ of the cases and smooth muscle layer in $87.5 \%$ of the animals. In the referential areas, that is, the areas adjacent to the site of the graft, urothelium was seen in $92.86 \%$, collagen in $42.86 \%$, and smooth muscle in $71.43 \%$ of the cases. In the control ureter (left ureter), urothelium and smooth muscle were seen in $100 \%$ of the animals, and collagen in $11.11 \%$. Comparing the 3 studied areas of the right ureter; it was observed that the presence of urothelium and smooth muscle was similar. However, the presence of collagen was higher in the experimental area in relation to the adjacent referential areas and in relation to the control left ureter (Table-3).

Table 2 - Mean diameter of the studied areas.

\begin{tabular}{lccc}
\hline Ureter & N & Mean diameter & Standard Deviation \\
\hline Left ureter diameter (control ureter) & 9 & 0.49 & 0.13 \\
Right proximal diameter & 9 & 1.48 & 0.97 \\
Graft site diameter & 9 & 0.91 & 0.40 \\
Right distal diameter & 9 & 0.67 & \\
\hline Left ureter x proximal right ureter & $\mathrm{p}=0.0077 *$ & \\
Left ureter x graft site in the right ureter & $\mathrm{p}=0.0173 *$ & \\
Left ureter x distal right ureter & $\mathrm{p}=0.0519$ & \\
Proximal right ureter x graft site & $\mathrm{p}=0.1235$ & \\
Proximal right ureter x distal right ureter & $\mathrm{p}=0.0077 *$ & \\
Graft site x distal right ureter & $\mathrm{p}=0.1282$ & & \\
$*$ statistically significant & & &
\end{tabular}

Table 3 - Urothelium, collagen and smooth muscle areas (in percentage) in the studied sites.

\begin{tabular}{lcccc}
\hline & $\begin{array}{c}\text { Control } \\
\text { Area }\end{array}$ & $\begin{array}{c}\text { Referential } \\
\text { Area }\end{array}$ & $\begin{array}{c}\text { Experimental } \\
\text { Area }\end{array}$ & Statistical significance \\
\hline Urothelium & $100 \%$ & $92.86 \%$ & $100 \%$ & $\mathrm{p} \geq(0.05)$ \\
Smooth muscle & $100 \%$ & $71.43 \%$ & $87.50 \%$ & $\mathrm{p} \geq(0.05)$ \\
Collagen & $11.1 \%$ & $42.86 \%$ & $100 \%$ & $\begin{array}{c}\text { Control area } x \text { referential area } \mathrm{p} \geq 0.05 \\
\text { Control area } \mathrm{x} \text { experimental area } \mathrm{p}=0.0004 * \\
\text { Referential area x experimental area } \mathrm{p}=0.017 *\end{array}$ \\
\hline * statistically significant & &
\end{tabular}


Regarding the inflammatory process, we observed that the graft area showed a higher degree of inflammation when compared to the referential area and to the control ureter (left ureter) $(\mathrm{p}=0.0002)$. Table- 4 shows the analysis of the inflammatory parameters with their respective scores and the final average score.

The acute inflammatory reaction was not different among the 3 studied areas. Concerning edema, neutrophils and congestion that characterized the acute process, no significant statistical difference was observed.

Concerning chronic inflammatory reaction, the concentration of granulation tissue was significantly higher in the graft area when compared to the control ureter $(\mathrm{p}=0.0078)$. The fibrosis was greater in the experimental area when compared to the referential and control area $(p=0.0156$ and $p=$ 0.0039 , respectively). No statistically significant difference was observed in the referential area when compared to the control area. Concerning monocytes, their concentration was significantly higher in the graft area and in the referential area when compared to the control ureter $(p=0.0039)$, however, no statistically significant difference was noted in the graft area when compared to the referential area.

The morphometric analysis of the specimens stained with the Sirius red was performed in order to assess the percentage of area occupied by smooth muscle and collagen (Table-5). We observed that the

Table 4 - Analysis of the inflammatory parameters with their respective scores and the final average score $(C=$ control ureter, $R=$ referential area, $E=$ experimental area)

\begin{tabular}{|c|c|c|c|c|c|c|c|c|c|c|c|c|c|c|c|c|c|c|c|c|c|}
\hline \multirow{2}{*}{$\begin{array}{r}\text { Pig } \\
\mathbf{N}^{\circ}\end{array}$} & \multicolumn{3}{|c|}{ Congestion } & \multicolumn{3}{|c|}{ Edema } & \multicolumn{3}{|c|}{ Neutrophils } & \multicolumn{3}{|c|}{ Granulation } & \multicolumn{3}{|c|}{ Fibrosis } & \multicolumn{3}{|c|}{ Monocytes } & \multicolumn{3}{|c|}{ Total } \\
\hline & $\mathbf{C}$ & $\mathbf{R}$ & $\mathbf{E}$ & $\mathbf{C}$ & $\mathbf{R}$ & $\mathbf{E}$ & $\mathbf{C}$ & $\mathbf{R}$ & $\mathbf{E}$ & $\mathbf{C}$ & $\mathbf{R}$ & $\mathbf{E}$ & $\mathbf{C}$ & $\mathbf{R}$ & $\mathbf{E}$ & C & $\mathbf{R}$ & $\mathbf{E}$ & $\mathbf{C}$ & $\mathbf{R}$ & $\mathbf{E}$ \\
\hline 1 & 0 & -1 & -1 & -1 & -1 & -1 & 0 & -1 & -1 & 0 & 1 & 3 & 0 & 1 & 3 & 0 & 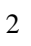 & 3 & -1 & 1 & 6 \\
\hline 2 & 0 & 0 & -1 & -1 & -1 & -2 & 0 & -1 & -1 & 0 & 2 & 2 & 0 & 1 & 3 & 0 & 3 & 3 & -1 & 4 & 5 \\
\hline 3 & 0 & 0 & -1 & -1 & -1 & -2 & 0 & -2 & -1 & 1 & 1 & 3 & 0 & 1 & 3 & 0 & 3 & 2 & 0 & 2 & 4 \\
\hline 4 & -1 & -3 & -1 & -2 & -3 & -1 & 0 & -1 & -1 & 0 & 2 & 2 & 0 & 1 & 2 & 0 & 3 & 3 & -3 & -1 & 4 \\
\hline 5 & 0 & 0 & -1 & -1 & -1 & -2 & 0 & 0 & -1 & 0 & 0 & 3 & 0 & 0 & 2 & 0 & 0 & 3 & -1 & -1 & 4 \\
\hline 6 & 0 & -1 & -1 & -1 & -1 & -1 & 0 & -1 & -1 & 1 & 1 & 2 & 0 & 0 & 3 & 0 & 3 & 3 & 0 & 1 & 5 \\
\hline 7 & 0 & -1 & -1 & -1 & -1 & -1 & 0 & -1 & -1 & 1 & 1 & 2 & 0 & 1 & 3 & 0 & 3 & 3 & 0 & 2 & 5 \\
\hline 8 & 0 & -1 & -1 & 0 & -2 & -2 & 0 & -1 & -1 & 1 & 1 & 2 & 0 & 3 & 3 & 0 & 2 & 3 & 1 & 2 & 4 \\
\hline 9 & -1 & -2 & -1 & -2 & -2 & -1 & 0 & -2 & -1 & 1 & 2 & 1 & 1 & 1 & 2 & 0 & 3 & 2 & -1 & 0 & 1 \\
\hline
\end{tabular}

Table 5 - Percentage of collagen and smooth muscle in the studied areas. (mean \pm standard deviation)

\begin{tabular}{|c|c|c|c|c|}
\hline & Control & $\begin{array}{l}\text { Referential } \\
\text { Area }\end{array}$ & $\begin{array}{l}\text { Experimental } \\
\text { Area }\end{array}$ & \\
\hline Muscle & $77.10 \pm 7.50$ & $70.94 \pm 11.66$ & $50.55 \pm 14.78$ & $\begin{array}{l}\text { Control } \mathrm{x} \text { Area of the experiment } \mathrm{p}=0.0002 * \\
\text { Referential area } \mathrm{x} \text { experiment area } \mathrm{p}=0.0027 *\end{array}$ \\
\hline Collagen & $22.74 \pm 7.50$ & $29.05 \pm 11.66$ & $49.443 \pm 14.79$ & $\begin{array}{l}\text { Control } \mathrm{x} \text { experiment area } \mathrm{p}=0.0002 * \\
\text { Referential area } \mathrm{x} \text { experiment area } \mathrm{p}=0.0027 *\end{array}$ \\
\hline
\end{tabular}

* statistically significant 
presence of smooth muscle was significantly higher in the control area and referential area, when compared to the experimental area. Regarding the amount of collagen in the studied areas, we could demonstrate that the experimental area showed a higher percentage of collagen in relation to the other 2 areas.

The histological study of the right kidneys demonstrates congestion in $55.55 \%$, pelvic edema in $66.66 \%$ and interstitial nephritis in $77.78 \%$ of the specimens. The hydronephrosis was present in $33.33 \%$ and the chronic pyelonephritis in $44 \%$ of the right kidneys. In one animal, we could observe the total absence of glomerulus in the renal parenchyma. The left kidney showed interstitial nephritis and congestion in $11.11 \%$ of the kidneys and pelvic edema in $33.33 \%$ of the specimens.

\section{COMMENTS}

The ideal biomaterial for urinary tract reconstruction would allow regeneration of tissue and maintenance of its function (1). Unfortunately, the reconstruction of a long segment of ureter is always a challenge for the surgeon. Tubular and non-tubular organs have been tried with limited success. The small intestine submucosa (SIS) has been used in different organs and structures such as the lower urinary tract, abdominal wall, bone, tendons, bladder, small bowel and skin $(1,10,11,24-26)$, with great success regarding tissue regeneration. When implanted in such structures it becomes indistinguishable from the host tissue. The biomaterial is remodeled by the host into replacement tissue with site-specific structural and functional properties. When implanted in a bladder site with pressurized static flow, SIS was remodeled into a tissue with measurable contractile activity. Jaffe et al. using this material in the reconstruction of the ureter in rabbits, verified that 3 native muscular layers of the ureter appeared in the collagen matrix of the grafted submucosa (11). The mechanisms of tissue remodeling are not yet completely understood. It has been shown that host cells proliferate and differentiate into site-specific tissue structures. Angiogenesis, cell migration, differentiation and deposition of extracellular matrix have been associated with this regenerative process. The proteoglycans and glycoproteins contain specific sites on their protein portion that help cells to attach and settle within the matrix. Another component of the matrix is the growth factors that stimulate growth and cell division, migration and differentiation.

The animal model chosen in this study was the pig, since its ureter presents a suitable diameter for the anastomosis and morphologically has a great similarity to the human ureter.

At the beginning of the study, we tried to implant the SIS in the canine ureter, but we had technical difficulties due to its small diameter and rigidity of its wall.

The urinary tract was evaluated on the 30th post-operative day in all animals, when they were submitted to ultrasound examination. The renal pelvis was dilated in $22.2 \%$ of the animals when compared to the left renal pelvis. In this study, the ultrasound did not appear to be a reliable examination. The great amount of gazes in the swine bowels did not allow a good visualization of the ureters.

All animals were sacrificed on the 40th postoperative day in order to assess surgical complications (abscess, fistula, incrustation), to measure the ureters diameter and to get the specimens for histological analysis. One pig died due to urinary fistula and 2 pigs developed hydronephrosis due to stenosis in the area of the SIS implantation.

Liatsikos et al. (9) used a 5F double-J stent to secure patency of the ureter. Probably if we had used the stent, the strictures in 2 animals could be avoided, but we must keep in mind that the stents can cause reflux to the kidney when the bladder contracts.

Concerning the ureters, we could verify that the right ureter showed significantly greater diameters in all the studied areas. It is important to note that when we compared the proximal right ureter and the site of the SIS implantation there was no statistically significant difference. This suggests that apart from the 2 animals mentioned above, SIS implantation did not determine important stenosis. The most important aspect of ureteral replacement is maintaining the normal drainage of the kidney, which requires the recovery of peristaltic activity in the ureter. Naturally, to have this activity smooth muscles are necessary. 
On the 40th postoperative day, our histological studies showed that muscular regeneration was present in $50 \%$ of the implanted area. In the referential areas, the muscle was present in $70.94 \%$ of the studied areas and in $77.1 \%$ of the control left ureter. Probably, if we had sacrificed the animals after a 3 months period or longer, we would find a greater percentage of muscles.

The consequences of a lesser concentration of smooth muscle and lack of innervation could explain the right ureteral enlargement comparing to the control left ureter. In the present study, SIS demonstrated to have a special characteristic of differentiation in the experimental area. There was evidence of urothelium regeneration along the ureteral segments implanted with SIS, supported by a collagen and smooth muscle background. Liatsikos et al. (9) observed urothelium regeneration in the $7 \mathrm{~cm}$ length of the SIS graft. They believe that this urothelial regeneration was because they maintained one third of the ureteral diameter. In spite of the fact that we maintained the same segment of the ureter, we do not believe that this could induce urothelial regeneration. The urothelium lining depends on the adjacent urothelium, besides the existence of a local neovascularization. Further studies, with total removal of the ureteral segment must be done in order to evaluate this property of the SIS membrane.

Regarding the inflammatory process, we did not observe acute inflammatory reaction. A chronic moderated inflammatory reaction was present in the graft area of the ureter; however, this reaction was not statistically different from the adjacent referential areas. In addition, a foreign body type reaction was not seen in the graft area.

We concluded that SID evolved in the grafted area to a chronic inflammatory tissue and high amount of muscular tissue, acting as a biologic scaffold, allowing the regeneration of the urothelium and the deposition of the collagen necessary for the repair of the ureteral injuries.

\section{REFERENCES}

1. Xie H, Shaffer BS, Wadia Y, Gregory KW: Use of reconstructed small intestine submucosa for urinary tract replacement. Asaio J. 2000; 46: 268-72.

2. Gallentine ML, Harmon WJ: Ureteral substitution with a stapled neoureter: a simplified Boari flap. J Urol. 2001; 166: 1869-72.

3. Fry DE, Milholen L, Harbrecht PJ: Iatrogenic ureteral injury. Options in management. Arch Surg. 1983; 118: 454-7.

4. Png JC, Chapple CR: Principles in ureteric reconstruction. Curr Opin Urol. 2000; 10: 207-12.

5. Desgrandchamps F: Endoscopic and surgical repair of the ureter. Curr Opin Urol. 2001; 11: 271-4.

6. Sacks MS, Gloeckner DC: Quantification of the fiber architecture and biaxial mechanical behavior of porcine intestinal submucosa. J Biomed Mater Res. 1999; 46: $1-10$.

7. Gloeckner DC, Sacks MS, Billiar KL, Bachrach N: Mechanical evaluation and design of a multilayered collagenous repair biomaterial. J Biomed Mater Res. 2000; 52: 365-73.

8. Baltaci S, Ozer G, Ozer E, Soygur T, Besalti O, Anafarta K: Failure of ureteral replacement with Goretex tube grafts. Urology 1998; 51: 400-3.

9. Liatsikos EN, Dinlenc CZ, Kapoor R, Bernardo NO, Pikhasov D, Anderson AE et al.: Ureteral reconstruction: small intestine submucosa for the management of strictures and defects of the upper third of the ureter. J Urol. 2001; 165: 1719-23.

10. Grossklaus DJ, Shappel SB, Adams MC, Brock JW.III, Pope JC.IV: Small intestine submucosa as a urethral coverage layer. J Urol. 2001; 166: 636-9.

11. Jaffe JS, Ginsberg PC, Yanoshak SJ, Costa LE Jr, Ogbolu FN, Moyer CP, et al.: Ureteral segment replacement using a circumferential small intestinal submucosa xenogenic graft. J Invest Surg. 2001; 14: 259-65.

12. Allman AJ, Mcpherson TB, Badylak SF, Merrill LC, Bhaskar K, Sheehan C, et al.: Xenogenic extracelular matrix grafts elicit a Th2-restriced immune response. Transplantation. 2001; 71: 1631-40.

13. Allman AJ, Mcpherson TB, Merrill LC, Badylak SF, Metzger DW: The Th2-restricted immune response to xenogeneic small intestine submucosa does not influence systemic protective immunity to viral and bacterial pathogens. Tissue Eng. 2002; 8: 53-62.

14. Byung-Soo K, Baez CE, Atala A: Biomaterials for tissue engineering. World J Urol. 2000; 18: 2-9. 
15. Badylak SF, Lantz GC, Coffey A, Geddes LA: Small intestinal submucosa as a large diameter vascular graft in dog. J Surg Res. 1989; 47: 74-80.

16. Badylak SF, Kropp B, Mcperson T, Liang H, Snyder PW. Small intestine submucosa: a rapidly resorbed bioscaffold for augmentation cystoplasty in a dog model. Tissue Eng. 1998; 4: 379-87.

17. Badylak SF, Voytik SL, Konini K, Shelbourne KD, Klootwyk T, Voytik SL, et al.: The use of xenogenic small intestinal submucosa as a biomaterial for Achilles' tendon repair in a dog model. J Biomed Mat Res. 1995; 29:977-85.

18. Roeder RA, Wolfe J, Lianakis N, Hinson T, Geddes LA, Obermiller J: Compliance, elastic modulus and burst pressure of small intestine submucosa (SIS), small diameter vascular grafts. J Biomed Mater Res. 1999; 47: 65-70.

19. Roeder RA, Lantz GC, Geddes LA: Mechanical remodeling of small-intestine submucosa smalldiameter vascular grafts - a preliminary report. Biomed Instrum Technol. 2001; 35: 110-20.

20. Brown-Etris M, Cutshall WD, Hiles MC: A new biomaterial derived from small intestine submucosa and developed into a wound matrix device. Wounds. 2002; 14: 150-66.

21. Hodde JP, Badylak SF, Brightman AO, Voytik-Harbin
SL: Glycosaminoglycans content of small intestine submucosa: a bioscaffold for tissue replacement. Tissue Eng. 1996; 2: 209-17.

22. Hurst RE, Bonner RB: Mapping of the distribution of significant proteins and proteoglycans in small intestinal submucosa by fluorescence microscopy. Journal of Biomaterial science. Polymer edition. 2001; 12: 1267-79.

23. Voytik-Harbin SL, Brightman AO, Kraine MR, Waisner B, Badylak SF: Identification of extractable growth factors from small intestinal submucosa. J Cell Biochem. 1997; 67: 478-91.

24. Prevel CD, Eppley BL, Sumerlin DJ, Jackson JR, McCarty M, Badylak SF: Small intestinal submucosa: utilization for repair of rodent abdominal wall defects. Ann Plast Surg. 1995; 35: 374-80.

25. Cook JL, Tomlinson JL, Arnoczky SP, Fox DB, Reeves CC, Kreeger JM: Kinetic study of the replacement of porcine small intestinal submucosa grafts and the regeneration of meniscal-like tissue in large avascular meniscal defects in dogs. Tissue Eng. 2001; 7: 321-34.

26. Dejardin LM, Arnoczky SP, Ewers BJ, Haut RC, Clarke RB: Tissue-engineered rotator cuff tendon using porcine small intestine submucosa. Histologic and mechanical evaluation in dogs. Am J Sports Med. 2001; 29: $175-84$.
Received: March 24, 2004

Accepted after revision: June 6, 2004

\author{
Correspondence address: \\ Dr. Fernando Hintz Greca \\ Av. Visconde de Guarapuava 5087 / 1401 \\ Curitiba, PR, 80240-010, Brazil \\ Fax: + 5541 242-6382 \\ E-mail: fernando.greca@bbs2.sul.com.br
}




\section{EDITORIAL COMMENT}

This is a very interesting article even though the number of animals studied is small and especially due to the short time frame analyzed. I am sure that if a third or forth group had been studied with 90 or 120 days we would certainly have more relevant information.

The authors have informed that they have used an ureteral catheter, apparently to model, during the surgery but they do not mention if this catheter has been withdrawn or not. In the discussion it is said that they did not used a double $\mathrm{J}$ with a fear of vesico- ureteral reflux. This is irrelevant, because we know that the use of these catheters is not harmful for a short period of time, either in animals or human beings. If this experiment was made in female animals it could allow the withdrawing of those catheters in the desired period using a common cystoscope.

Finally, I believe that this material, that is being already used with other objectives could also be used in this field. I believe that further studies are needed with a more appropriate methodology aiming at solidifying the results presented here.

Dr. Salvador Vilar C. Lima Section of Urology, Federal University of Pernambuco Recife, PE, Brazil 